Textures and Microstructures, Vol. 34, pp. 91-103 Reprints available directly from the publisher Photocopying permitted by license only
(C) 2000 OPA (Overseas Publishers Association) N.V.

Published by license under the Gordon and Breach Science Publishers imprint. Printed in Malaysia.

\title{
IN-PLANE TEXTURING IN SPUTTERED FILMS
}

\author{
J.F. WHITACRE*, S.M. YALISOVE and J.C. BILELLO \\ University of Michigan, Department of Materials Science and \\ Engineering, 2300 Hayward Street, Ann Arbor, MI 48109, USA
}

(Received 8 October 1999)

Films consisting of $\mathrm{Mo}, \mathrm{Cr}$, and $\mathrm{Ta}$ have all been found to display well-defined biaxial textures when grown under certain conditions. A well-defined out-of-plane texture evolves within the first $\sim 100 \mathrm{~nm}$ of the film, followed by the evolution of a preferred crystallographic orientation in the plane of the film. These effect were studied using X-ray polefigure analysis, scanning electron microscopy (SEM), transmission electron microscopy (TEM), transmission electron diffraction (TED), and high resolution grazing incidence X-ray scattering (GIXS). It has been found that in-plane texture evolves only when there is, on average, oblique adatom flux incident onto the substrate. Further, the type of outof-plane texture can be controlled by altering the deposition conditions. Parameters including cathode-to-substrate distance, deposition rate, average angle of adatom incidence, and sputter gas pressure, have been shown to determine the type out-of-plane texture, as well as the rate of in-plane texture evolution. The studies conducted have shown that it is possible to create and control biaxially textured films and multilayers made of a variety of materials. A recent model which describes this phenomena is discussed.

Keywords: Sputtered films; Texture; Multilayers; X-ray; TEM; SEM

\section{INTRODUCTION}

Thin metallic films are frequently used in applications such as integrated circuit interconnects (Muraka, 1993), protective coatings (Windischmann, 1992; Adams et al., 1993), magnetic recording media (Katoaka et al., 1993; Kief and Egelhoff Jr., 1993; Kim et al., 1993), and superconducting multilayers (Mattson, 1997; Bauer et al., 1997).

\footnotetext{
* Corresponding author.
} 
The texturing in these films is a critical property, and the ability to control the development of both out-of-plane and in-plane texture is of interest. Relevant studies have focused on the effects of energetic ion beams (Bradley et al., 1986), sputter gas pressure (Je et al., 1997), deposition geometry (Hagemeyer et al., 1993; Karpenko et al., 1994; Bilello et al., 1995; Harper et al., 1997; Rodriguez-Navarro et al., 1998), surface energetics (Knuyt et al., 1995; Thompson and Carel, 1995), and impurity concentration (van de Waterbeemd and van Oosterhout, 1967) have on texturing. The development of in-plane texture has been attributed to a number of mechanisms. The existence of oblique adatom flux has been found to be necessary in some cases (Karpenko et al., 1994; Harper et al., 1997; Bauer et al., 1997). Others used an off-axis ion beam to preferentially sputter films during growth, thereby encouraging the formation of in-plane texture (Bradley et al., 1986). Still another investigation has shown that films grown without any average oblique influence do not develop any in-plane texturing (Malhotra et al., 1996). Deposition geometry plays a critical role in in-plane texturing, while the role of deposition kinetics and energetics are not currently well understood.

Below is a review of work done to increase understanding of texture evolution in thin sputtered films. A particular set of deposition conditions which lead to the formation of a strong biaxial texture in sputtered films of Mo, $\mathrm{Cr}, \mathrm{CrN}$, and Ta will be described. Once the basic phenomena of in-plane texturing was well documented, further experiments were done to provide better understanding of the role of adatom kinetics during growth. This was accomplished by altering the sputter gas pressure, chamber geometry, deposition rate, and growth temperature during film growth. Experimental results will be presented and discussed in light of a model which describes in-plane texturing and is based on two simple physical mechanisms.

\section{DESCRIPTION OF EXPERIMENTS}

\section{Deposition Chamber}

All films were deposited in a Denton ${ }^{\mathrm{TM}}$ cryo-pumped DC magnetron sputter chamber (Whitacre et al., 1998). A base pressure of at most $3 \times 10^{-6}$ Torr was used, and the pressure of Ar sputter gas could be 
varied from 2 to $20 \mathrm{mT}$ Torr. The substrates, as received test-grade $\mathrm{Si}$ (100) wafers, were mounted on a platen which rotated beneath the sputter target at a rate of $20 \mathrm{rpm}$. This setup allowed for multiple films to be grown at once. The substrates were exposed to a full $180^{\circ}$ range of flux in the plane parallel to their direction of rotation. In the plane normal to rotation direction, however, the angular flux range is much less. This configuration provides a break in the symmetry of the deposition: there is, on average, more oblique flux in one plane over any other. Figure 1 shows a schematic of the deposition chamber.

\section{Analysis Techniques}

Microstructure and film texturing were studied using scanning electron microscopy (SEM), transmission electron microscopy (TEM), transmission electron diffraction (TED), X-ray pole figure analysis, and high resolution X-ray diffraction (using the symmetric GIXS geometry). An Hitachi S-800 field emission SEM was used with its accelerating potential at $7 \mathrm{kV}$ and its sample distance set to $5 \mathrm{~mm}$, allowing for resolved images to be recorded at magnifications of 20,000-40,000x. Further analyses used a JEOL 2000 TEM (accelerating voltage $=$ $200 \mathrm{kV}$ ). Plan-view samples were created using an $\mathrm{HF}$ etching technique (Whitacre et al., 1998), while cross section samples were made by tripod polishing. TED was performed using the JEOL 2000 to qualitatively analyze texturing. All diffraction patterns were recorded from a defined area of equal size in each of the samples using a selected area aperture.

Pole figure analysis was done using both a Rigaku reflection pole figure apparatus which used the Schultz geometry, and an inel ${ }^{\mathrm{TM}}$

(a)

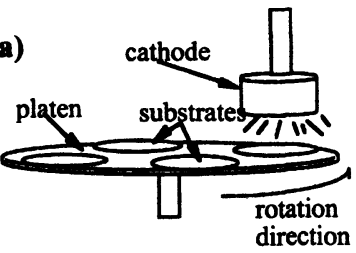

(b)

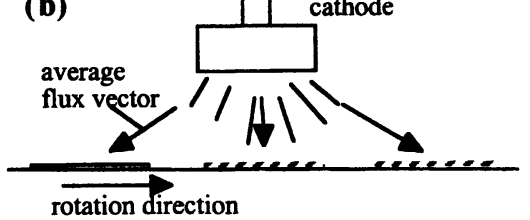

FIGURE 1 Schematic showing deposition chamber geometry: (a) overview, and (b) representation showing how the average flux vector changes angle of incidence upon the substrate during rotation. 
position sensitive detector attached to a Huber four-circle diffractometer (Karpenko et al., 1994; Malhotra et al., 1998).

$\phi$ scans collected in the symmetric grazing incidence $X$-ray scattering (GIXS) geometry allowed for the degree of in-plane texturing of films to be studied precisely at varying depths (Karpenko, 1996).

\section{RESULTS AND DISCUSSION}

\section{Texture Evolution in Mo Films}

The evolution of both in-plane and out-of-plane textures was observed in Mo films grown at a rate of $34 \mathrm{~nm} / \mathrm{min}$ under the conditions described above (Karpenko et al., 1994; Bilello et al., 1995). Texture evolution may be seen in the (110) pole figures and corresponding TEM/TED analysis shown in Fig. 2.

By the time the film reaches a thickness of $200 \mathrm{~nm}$, a well-defined outof-plane texture, (110) in this case, is observed. As the films become thicker, both the out-of-plane and in-plane textures become stronger. The $2 \mu \mathrm{m}$ thick film displays a "times random" value of 16 : this film is 16 times more organized crystallographically than a randomly oriented sample (Karpenko et al., 1994). The TEM/TED analysis supports the pole figure data: as the film becomes thicker, the electron diffraction

(a)

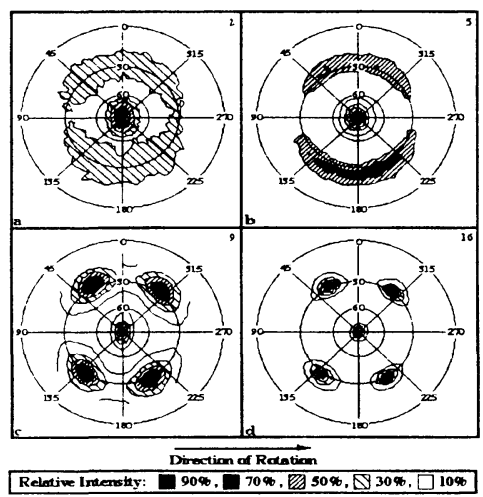

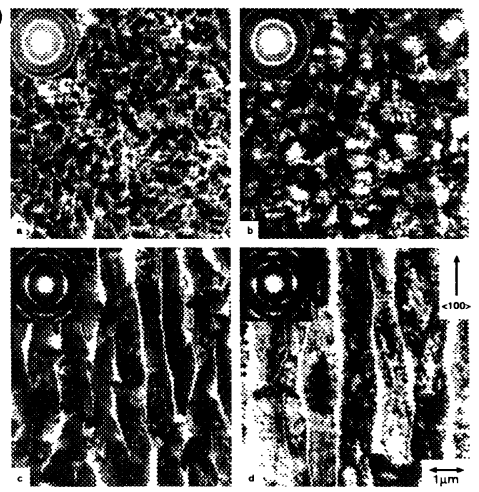

FIGURE 2 (a) Pole figures showing the evolution of texture in Mo films for thicknesses of a: 200 run, b: 500 run, c: 1000 run and d: 2000 run. The number in the upper right hand corner of each pole figure is the "times random" value. (b) TEM/TED analysis for the same films. The direction of grain elongation in the thicker films is normal to direction of rotation during growth (figure adapted from Karpenko et al., 1994). 
patterns show an increase in localized diffraction consistent with the existence of in-plane texture. There is also an increase in grain size in the plane of the film; the grains become elongated in the direction normal to substrate motion. By comparing the electron diffraction patterns to the bright field TEM images, it can be deduced that the grains are elongated in their (100) direction and are narrowest in the (110) direction. The surface morphology of the $2 \mu \mathrm{m}$ thick film is shown in the SEM, AFM, and cross section TEM images of Fig. 3. The grains are faceted and have some void formation between them, as attested to by the TEM image.

$\mathrm{X}$-ray analysis, $\phi$ scans in the symmetric GIXS geometry, of Mo films of different thicknesses show the evolution of in-plane texturing (Karpenko, 1996). Figure 4 has this data. The full-width at halfmaximum of the recorded peaks is inversely related to the degree of in-plane texturing; the diffracted intensity at any $\phi$ is proportional to the number of grains with that particular in-plane orientation. This type of analysis allows for precise comparison of the degree of in-plane texturing in various films.

\section{Chromium}

Cr films grown under nominally the same conditions as the Mo films described above also displayed in-plane texturing. Figure 5 shows the

(a)

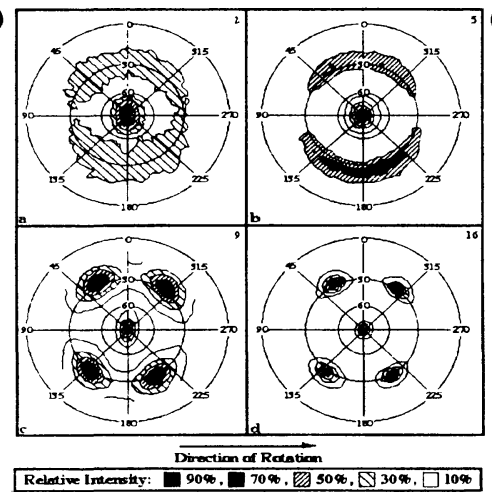

(b)

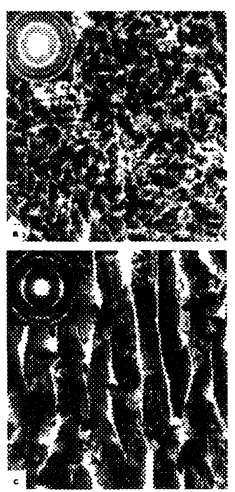

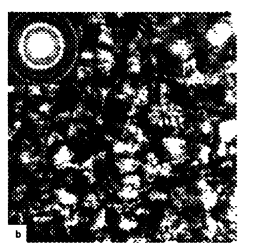

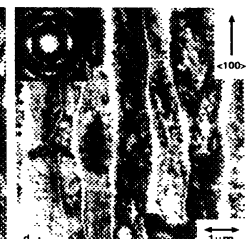

FIGURE 3 Comparative images showing the surface morphology of a $2 \mu \mathrm{m}$ thick Mo Film: (a) plan-view SEM, (b) AFM (the profile plot is a cross section taken from the area indicated by the white line), and (c) cross section bright-field TEM (figure adapted from Whitacre et al., 1998). 


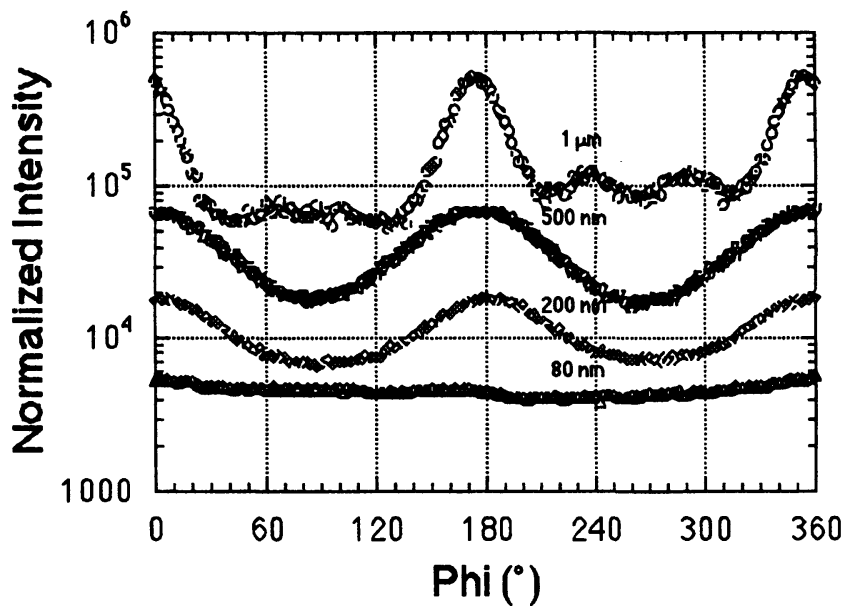

FIGURE 4 Plot showing the detected diffracted intensity in for a textured Mo film oriented in the (110) Bragg condition using $\phi$ scans in the symmetric GIXS geometry. For each film, the X-ray penetration depth was $100 \mathrm{~nm}$. The thicker the film, the higher the degree of texture observed in the top 100 run (figure adapted from Karpenko, 1996).
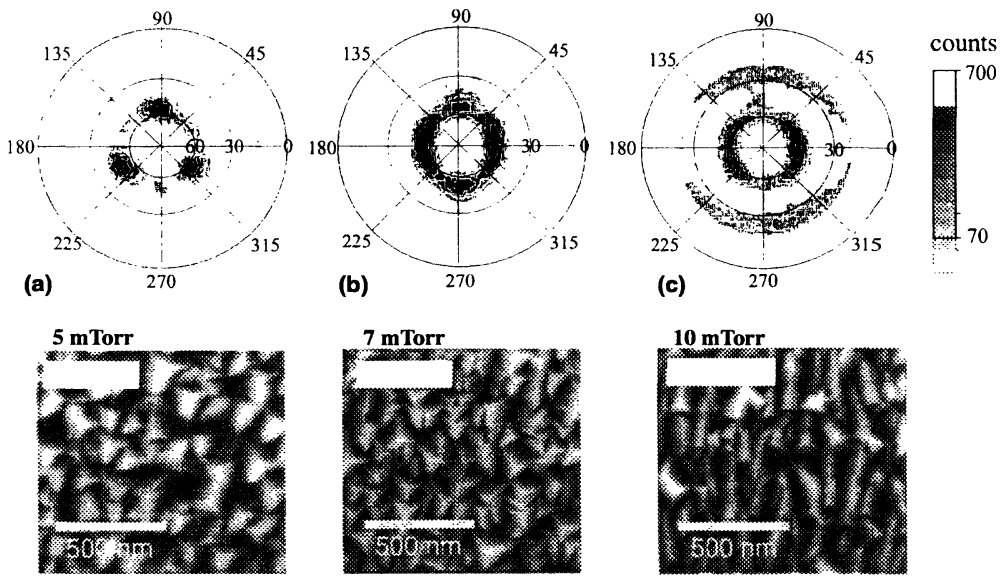

FIGURE 5 Plan-view SEM images and corresponding (110) pole figures for $1.5 \mu \mathrm{m}$ thick Cr films grown with Ar sputter gas pressures of (a) $5 \mathrm{mTorr}$, (b) $7 \mathrm{mT}$ orr, and (c) $10 \mathrm{mT}$ Torr. The $5 \mathrm{mT}$ Torr film has a strong (111) out-of-plane texture and a welldefined in-plane texture. The in-plane texture is less defined in the 7 and $10 \mathrm{mTorr}$ cases, where the dominant our of plane texture is (112). 
surface morphology and (110) pole figures from a $\mathrm{Cr}$ film grown at room temperature with a cathode height of $9.5 \mathrm{~cm}$, a rate of $\sim 20 \mathrm{~nm} /$ min and three different Ar pressures: 5, 7 and $10 \mathrm{mTorr}$. The pole figures show that in-plane texture occurs in varying degrees in $\mathrm{Cr}$ films. Texturing dependence on sputter gas pressure will be discussed in a later section. Though the grain shape and symmetry of the $\mathrm{Cr}$ films are not identical to those observed in Mo, the same effects and evident: grain elongation in the plane of growth normal to the direction of substrate motion during growth, and faceted surface morphology.

\section{Tantalum}

Figure 6 shows the surface morphology and corresponding (002) and (202) pole figures of a $3 \mu \mathrm{m}$ thick $\beta$ (tetragonal phase) Ta film grown under the conditions described in the experimental section. The microstructure differs dramatically from that observed in the Mo and $\mathrm{Cr}$ films; the surface is not faceted, but instead consists of elongated rounded features. As in the other cases, however, the direction of feature elongation is normal to the direction of substrate motion during deposition. The pole figures show that the film has a strong (002) outof-plane texture. The (202) pole figure shows that there is a significant in-plane texture, though it is not as strong as that observed in films such as Mo or Cr.

\section{Chromium Nitride}

In-plane texturing has been observed in $\mathrm{CrN}$ (Zhao, 1998). The deposition conditions leading to these effects are identical to those described

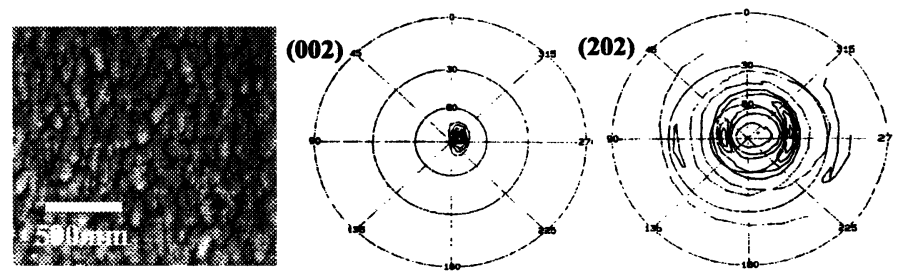

FIGURE 6 Plan-view SEM images and corresponding pole figures for $3 \mu \mathrm{m}$ thick Ta film. The pole figures indicate a strong (002) out-of-plane texture and some inplane texture. The direction of surface feature elongation is normal to the direction of substrate motion during deposition. 
above, except that now the reactive sputtering is used. A dependence of nitrogen content on texturing was observed.

\section{Texture Control}

The type of out-of-plane texture has been found to depend upon the cathode height during deposition, the growth rate (Karpenko et al., 1994; Bilello et al., 1995), and sputter gas pressure (Windischmann, 1992). Figure 7 shows SEM and corresponding (110) pole figures for $2.5 \mu \mathrm{m}$ Mo films grown with a cathode height of 9.5 and $12 \mathrm{~cm}$. If the cathode height is $9.5 \mathrm{~cm}$, there is a strong 111 out-of-plane texture component, while the film grown with the cathode height at $12 \mathrm{~cm}$ shows only (110) type out-of-plane texture. These films were grown at, nominally, the same rate. If the rates are changed, however, we also see a difference in the texture orientation (Karpenko et al., 1994). There is a difference between $2 \mu \mathrm{m}$ thick Mo films grown at different growth rates: films grown at $68 \mathrm{~nm} / \mathrm{min}$ displays a strong (111) type out-ofplane orientation, while similar films grown at $34 \mathrm{~nm} / \mathrm{min}$ shows a strong (110) out-of-plane texture.

Figure 5 shows pole figures from $\mathrm{Cr}$ films grown at three different $\mathrm{Ar}$ pressures: 5,7 , and $10 \mathrm{~m}$ Torr. The type of out-of-plane texture depends upon the sputter gas pressure. The film grown at $5 \mathrm{mTorr}$ has a (111) out-of-plane texture, while the film grown at $10 \mathrm{~m}$ Torr displays a (112) out-of-plane texture. The $7 \mathrm{mTorr}$ film has a mix of the two types of out-of-plane texturing. The film deposited using $5 \mathrm{mT}$ Trr of Ar displays a higher degree of in-plane texture than the films grown under 7 and $10 \mathrm{mTorr}$ of Ar.
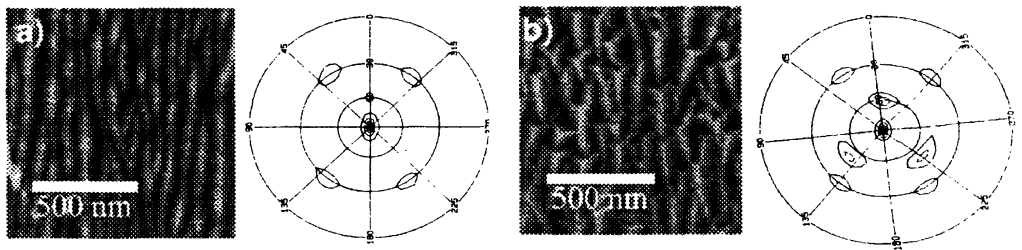

FIGURE 7 Plan-view SEM images and corresponding (110) pole figures for $2 \mu \mathrm{m}$ thick Mo films grown with cathode-to-platen distances of (a) $11 \mathrm{~cm}$ and (b) $7 \mathrm{~cm}$. The smaller cathode to substrate distance provided a mixed (111)/(110) out-of-plane texture. 
(a)

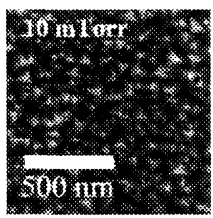

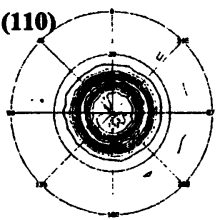

(b)
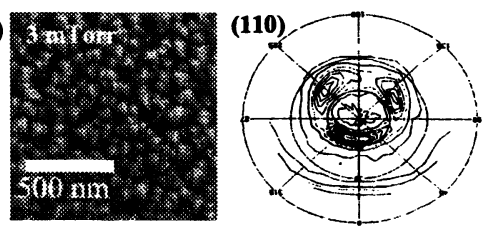

FIGURE 8 Plan-view SEM images and corresponding (110) pole figures for $1.5 \mu \mathrm{m}$ thick Cr films grown at $290^{\circ} \mathrm{C}$ with Ar pressures of (a) $10 \mathrm{mTorr}$, and (b) $3 \mathrm{mT}$ Torr. There is less in-plane organization for the film grown at $10 \mathrm{mT}$ Torr. Both films display a strong (111) out-of-plane texture.

\section{Temperature Effects}

Chromium films were deposited at a temperature of $\sim 290^{\circ} \mathrm{C}$ using two different sputtering gas (Ar) pressures. The cathode height was $9.5 \mathrm{~m}$ and a shielded filament heater affixed to the ceiling of the deposition chamber was used. Figure 8 shows plan-view SEM and (110) pole figures of two $2 \mu \mathrm{m}$ films grown using 10 and $3 \mathrm{mT}$ Torr of Ar.

Several differences from previous growths are evident. The grain size is significantly smaller than films grown under the same conditions at room temperature. The grains are still faceted, though they are no longer elongated in the plane of growth. The pole figures show that each film has a (111) out-of-plane texture, but only the film grown using the lower Ar pressure of $3 \mathrm{mT}$ Torr displays any in-plane texturing.

\section{Growth onto Rough Surfaces}

Mo films $400 \mu \mathrm{m}$ thick were deposited onto well-defined roughened surfaces (Whitacre et al., 1998). This study found that if the rough features were large enough to limit the angular range of adatom flux during growth there is a measurable decrease in the rate of in-plane texturing (Whitacre et al., 1998). By slightly manipulating the geometry of the deposition, substrate roughness limits the films texturing.

\section{Discussion}

It is evident that in-plane texture develops in thin sputtered films if there is: (1) on average, oblique adatom flux, and (2) sufficient adatom kinetic energy. 
If the substrate rests directly beneath the cathode during growth, no in-plane texture develops, though a strong out-of-plane texture and faceted surface morphology exists (Malhotra et al., 1996). If, however, the substrate is kept either at a stationary off-axis location (Malhotra et al., 1996) or is rotated beneath the cathode, biaxial texture is created in the film. It has been shown that if the substrate surface is roughened such that the average angle of oblique adatom flux is limited, the rate of in-plane texturing decreases (Whitacre et al., 1998). In all of these cases, an out-of-plane texture develops first, followed by the evolution of in-plane texture as grains compete during growth.

A model has been proposed which describes in-plane texturing (Karpenko et al., 1997). It is assumed that in-plane texturing is a result of the combination of two physical mechanisms: (1) anisotropic surface transport, and (2) atomistic shadowing effects which occur with oblique adatom incidence. Anisotropic surface transport causes grains to grow faster along a particular crystallographic direction in the plane of growth. For example, Mo grains grow most rapidly along their (100) axis and slowest in the (110) direction. This results in grains which are elongated (to increasing degrees as the films thicken) along their (100) direction.

If there is minimal surface diffusion, atomic shadowing occurs. Adatoms pile up on existing grains creating significant void formation between those grains (Dirks and Leams, 1977). If the adatoms are obliquely incident onto the substrate, this shadowing effect will also cause the adatom pile up to be more efficient in one in-plane direction over any other. In particular, adatoms will contribute most efficiently to in-plane formation in the direction normal to the projection of the flux vector onto the plane of the substrate. This is the in-plane direction of highest adatom capture efficiency. Minimum capture efficiency is in the direction parallel to the projection of the incoming flux vector. When both shadowing and anisotropic surface transport conditions exist concurrently with oblique adatom flux, those grains whose crystallographic fast in-plane growth direction corresponds to the highest adatom capture efficiency direction will compete favorably during deposition. Eventually, only those grains that nucleated with their fast crystallographic growth direction aligned with the direction of highest adatom capture efficiency due to shadowing will survive. This may be 
expressed mathematically and compared with X-ray data collected from films was in-plane texturing (Karpenko, 1996).

If this model is correct, an increase in either surface temperature or adatom kinetic energy should affect the in-plane texturing rate by altering anisotropic surface diffusion and the effectiveness of the shadowing mechanism. If the film surface is heated, surface diffusion should dominate the shadowing effect and in-plane texture should evolve more slowly. This is observed in the $\mathrm{Cr}$ films studied, where the film grown at $\sim 290^{\circ} \mathrm{C}$ in $10 \mathrm{mT}$ orr of $\mathrm{Ar}$ has almost no in-plane texturing compared to a similar film grown at room temperature.

An increase in adatom kinetic energy should promote surface diffusion and work against the shadowing effect. By lowering the Ar pressure, the average adatom kinetic energy increases (Meyer et al., 1981). Experimentally, different (or mixed) out-of-plane texturing is observed. However, there is not a significant difference in the degree of texturing between $\mathrm{Cr}$ films using different Ar pressures. This effect is currently under consideration and will be addressed in a later publication.

\section{CONCLUSIONS}

For sputtered thin films to develop a biaxial texture, there must be, on average, some off-axis or oblique geometrical influence on the growing film to allow for grain competition during growth. This paper has addressed how the presence of oblique adatom flux during DC magnetron sputtering leads to the development of in-plane texture. Once the general phenomena was characterized in a variety of films, further studies were done to explore the role of surface energetics and adatom kinetics during growth. It has been shown that in-plane grain competition is sensitive to surface diffusion; films develop in-plane texture more slowly at elevated temperatures. It has also been shown the an increase in adatom kinetic energy, while being enough to control the type of out-of-plane texture, is not enough to dramatically affet the rate of in-plane texturing. The relevance of these results to a model which describes the evolution of in-plane texturing was discussed. By addressing questions raised, a new level of understanding of in-plane texturing in sputtered films has been achieved. 


\section{Acknowledgments}

This work was supported under ARO Army contract numbers DAAH 04-95-1-0120 and DAAG 55-98-1-0382. Diffraction data collected at SSRL beam line 7-2, funded by the US DoE.

\section{References}

Adams, D.P., Vill, M., Tao, I., Bilello, J.C., and Yalisove, S.M. (1993). Controlling strength and toughness of multilayer films: A new multiscalar approach. Journal of Applied Physics, 74, 1015.

Bauer, M., Schwachulla, J., Furtner, S., Berberich, P., Kinder, H., Rogalla, H. and Blank, D.H.A. (1997). Deposition of YSZ, CeO/sub 2/, and $\mathrm{MgO}$ on amorphous and polycrystalline substrates. Proceedings of EUCAS 1997, 3rd European Conference on Applied Superconductivity, 2, 30.

Bilello, J.C., Yalisove, S.M. and Rek, Z.U. (1995). The evolution of texture in thin films and multilayers via synchrotron transmission Laue and grazing-incidence $\mathrm{X}$-ray scattering. Journal of Physics D: Applied Physics, 28, A295.

Bradley, R.M., Harper, J.M. and Smith, D.A. (1986). Theory of thin-film orientation by ion bombardment during deposition. Journal of Applied Physics, 60(12), 4160.

Dirks, A.G. and Leamy, H.J. (1977). Columnar microstructure in vapor-deposited thin films. Thin Solid Films, 47, 219-233.

Hagemeyer, H., Richter, H.J., Hibst, H., Maier, V. and Marosi, L. (1993). Crystallographic texture and morphology of obliquely deposited $\mathrm{Co}-\mathrm{Cr}$ magnetic thin films on flexible polymeric substrates. Thin Solid Films, $230,199$.

Harper, J.M.E., Rodbell, K.P., Colgan, E.G. and Hammond, R.H. (1997). In-plane crystallographic texture of BCC metal thin films on amorphous substrates. Materials Research Society Symposium Proceedings, Vol. 472: Polycrystalline Thin Films: Structure, Texture, Properties and Applications III, p. 27.

Je, J.H., Noh, D.Y., Kim, H.K. and Liang, K.S. (1997). Epitaxial and island growth of Ag/Si(001) by rf magnetron sputtering. Journal of Applied Physics, 81(9), 6126.

Katoaka, H., Bain, J.A., Brennan, S. and Clemins, B.M. (1993). Crystallographic anisotropy in thin film magnetic recording media analyzed with $\mathbf{X}$-ray diffraction. Journal of Applied Physics, 73(11), 7591.

Karpenko, O.P. (1996). Evolution of surface roughness and texture during low temperature film deposition. Ph.D. thesis, University of Michigan, pp. 87-101.

Karpenko, O.P., Bilello, J.C. and Yalisove, S.M. (1994). Combined transmission electron microscopy and $\mathrm{X}$-ray study of the microstructure and texture in sputtered Mo films. Journal of Applied Physics, 76(8), 4610.

Karpenko, O.P., Bilello, J.C. and Yalisove, S.M. (1997). Growth anisotropy and selfshadowing: a model for the development in in-plane texture during polycrystalline thin-film growth. Journal of Applied Physics, 82(3), 1397.

Kief, M.T. and Egelhoff, Jr., W.F. (1993). Perpendicular magnetic anisotropy in epitaxial ultrathin films of $\mathrm{Fe}$ and $\mathrm{Co}$ on $\mathrm{Cu}(100), \mathrm{Cu}(110)$ and $\mathrm{Cu}(111)$. Journal of Applied Physics, 73, 6195.

Kim, M.R., Guruswamy, S. and Johnson, K.E. (1993). Microstructural origin of in-plane magnetic anisotropy in magnetron in-line sputtered $\mathrm{CoPtCr}$ thin-film disks. Journal of Applied Physics, 74, pp. 4643.

Knuyt, G., Quaehaegens, C., D’Haen, J. and Stals, L.M. (1995). A quantitative model for the evolution from random orientation to a unique texture in PVD thin film growth. Thin Solid Films, 258, 159. 
Malhotra, A.K., Yalisove, S.M. and Bilello, J.C. (1996). Origin of in-plane texturing in sputtered Mo films. Materials Research Society Symposium Proceedings, 403, 33.

Malhotra, A.K., Whitacre, J.F., Zhao, Z.B., Hershberger, J., Yalisove, S.M. and Bilello, J.C. (1998). An in situ/ex sito X-ray analysis system for thin sputtered films. Surface and Coatings Technology, 110, 105-110.

Mattson, J.E. (1997). Properties of $\mathrm{Ni} / \mathrm{Nb}$ magnetic/superconducting multilayers. Journal of Vacuum Science and Technology A (Vacuum, Surfaces, and Films), 15(3), 1774-1779.

Meyer, K., Schuller, I.K. and Falco, C.M. (1981). Thermalization of sputtered atoms, Journal of Applied Physics, 52(9), 5803-5805.

Rodriguez-Navarro, A., Otano-Rivera, W. and Garcia-Ruiz, J.M. (1998). Control of the preferred orientation of A1N thin films by collimated sputtering. Journal of Vacuum Science and Technology A, 16(3), 1244.

Thompson, C.V. and Carel, R. (1995). Texture development in polycrystalline thin films. Materials Science and Engineering B, 32, 211.

van de Waterbeemd, J.G.W. and van Oosterhout, G.W. (1967). Impurity incorporation and texturing in thin films. Philips Research Report, 22, 375.

Windischmann, H. (1992). Stress in thin films. Critical Reviews in Solid State and Materials Science, 17, 547.

Whitacre, J.F., Rek, Z.U., Bilello, J.C. and Yalisove, S.M. (1998). Surface roughness and in-plane texturing in sputtered thin films. Journal of Applied Physics, 84(3), 1346.

Zhao, Z.B. (1998). Private communication with author, University of Michigan, Department of Materials Science and Engineering, August 1998. 